\title{
Magnetoelectric birefringence revisited
}

\author{
V. A. De Lorenci* \\ Institute of Science, Federal University of Itajubá, 37500-903 Itajubá, M. G., Brazil \\ and PH Department, TH Unit, CERN, 1211 Geneva 23, Switzerland \\ G. P. Goulart ${ }^{+}$ \\ Institute of Science, Federal University of Itajubá, 37500-903 Itajubá, M. G., Brazil
}

(Received 6 May 2008; published 15 August 2008)

\begin{abstract}
Electromagnetic wave propagation inside isotropic material media characterized by dielectric coefficients $\varepsilon_{\mu \nu}(E, B)$ and $\mu_{\mu \nu}(E, B)$ is examined. The regime of the eikonal approximation is considered. The Hadamard method of field disturbances is used and the dispersion relations are obtained by solving the Fresnel equation. Some applications of the formalism are presented. Particularly, birefringence phenomena induced by applied external fields are derived and discussed. It is shown that the magnetoelectric birefringence effect can occur even without the presence of Kerr and Cotton-Mouton effects, provided the physical system satisfies certain conditions.
\end{abstract}

DOI: 10.1103/PhysRevD.78.045015

PACS numbers: 42.25.Lc, 03.50.De, 04.20.- $-\mathrm{q}$

\section{INTRODUCTION}

Inside material media the Maxwell equations must be supplemented with constitutive relations between the applied external fields and their induced excitations. Such relations are generally nonlinear (linear constitutive relations have also been considered in the literature [1]) and depend on the physical properties of each considered medium under the action of external fields. A remarkable consequence coming from the nonlinearity of the field equations is the artificially induced birefringence phenomenon: waves with different polarization propagate with different velocities due to the presence of applied external fields [2,3]. In fact, the effect of applying an external field is just to induce an artificial optical axes, which in general disappears as soon as the external field is turned off. Hence, the initially isotropic medium becomes anisotropic under the action of external fields. In the context of crystalline systems the optical axes can be present in the medium and birefringence occurs naturally $[4,5]$. In this case, by applying an external field both effects (natural and induced birefringences) interfere, leading to a nontrivial mechanism of birefringence control by means of external electromagnetic fields [6].

In the regime of intense electromagnetic fields the Maxwell linear theory is no longer applicable and new effects, including birefringence, emerge from the quantum regime [7]. The analysis of light propagation shows that there is a non-null probability of photon splitting under a strong external electromagnetic field [8,9]. Investigations on light propagation in the context of nonlinear Lagrangian for electrodynamics can be found in [10-14].

Birefringence induced by external electromagnetic fields has been long ago reported in the literature [15-

\footnotetext{
*delorenci@unifei.edu.br

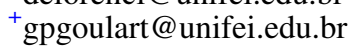

20]. Distinct proposals for its theoretical description were presented in [21-24], where some new aspects of this phenomenon were predicted. Among them, the existence of the so-called magnetoelectric birefringence was discussed, which is a kind of birefringence linear in the product of the electric and magnetic fields. The experimental observation of the magnetoelectric birefringence was recently reported in the literature [25,26].

Birefringence is nowadays widely used in the technology of optical devices as well as a technique for investigating properties of several physical systems, including astrophysical phenomena [27].

In this work, birefringence phenomena are investigated in the context of homogeneous dielectric media at rest with the dielectric coefficients $\varepsilon^{\mu}{ }_{\nu}=\varepsilon^{\mu}{ }_{\nu}(E, B)$ and $\mu^{\mu}{ }_{\nu}=$ $\mu^{\mu}{ }_{\nu}(E, B)$ in the limit of geometrical optics. The analysis is restricted to local electrodynamics, where dispersive effects are neglected. Only monochromatic waves are considered, thus avoiding ambiguities with the velocity of the wave.

In Sec. II the eigenvalue equation associated with the light propagation in general nondispersive material media is presented. In Sec. III the dispersion relations are obtained for isotropic media described by $\varepsilon^{\mu}{ }_{\nu}=\varepsilon^{\mu}{ }_{\nu}(E, B)$ and $\mu^{\mu}{ }_{\nu}=\mu^{\mu}{ }_{\nu}(E, B)$. Applications for birefringence phenomena are investigated in Sec. IV, where some particular cases are obtained from the formalism. The consequences of the anisotropy present in the propagation of the extraordinary ray are discussed. Some final remarks are presented in the conclusion section.

A Minkowskian spacetime employing a Cartesian coordinate system is used throughout this work. The background metric is denoted by $\eta_{\mu \nu}=\operatorname{diag}(+1,-1$, $-1,-1)$. All quantities are refereed as measured by the geodetic observer $V^{\mu}=c \delta_{0}^{\mu}$, where $\delta_{\nu}^{\mu}$ denotes the Kronecker tensor. For any quantity $X^{\mu}=(0, \vec{X})$ we define 
its modulus as $X \doteq\left(-X^{\mu} X_{\mu}\right)^{1 / 2}$ and the associated unit vector as $\hat{X} \doteq \vec{X} / X$. In the same way we use the notation $\hat{X}^{\mu} \doteq X^{\mu} / X$. For any two quantities $X^{\mu}$ and $Y^{\mu}$ we denote its scalar product $X^{\mu} Y_{\mu}$ as $(X Y)$. As an example, the electric field is represented by $E^{\mu}=(0, \vec{E})$, whose modulus is $E=\left(-E^{\alpha} E_{\alpha}\right)^{1 / 2}$. Similarly, the magnetic field is $B^{\mu}=(0, \vec{B})$. Its scalar product is given by $(E B)=$ $E^{\mu} B_{\mu}=-\vec{E} \cdot \vec{B}$. The units are such that $c=1$.

\section{EIGENVALUE EQUATION}

The electrodynamics in a continuum medium at rest is completely determined by the Maxwell equations [28]

$$
\begin{aligned}
& V^{\mu} D^{\alpha}{ }_{, \mu}+\eta^{\alpha \beta \gamma \delta} V_{\gamma} H_{\delta, \beta}=0, \\
& V^{\mu} B^{\alpha}{ }_{, \mu}-\eta^{\alpha \beta \gamma \delta} V_{\gamma} E_{\delta, \beta}=0,
\end{aligned}
$$

together with the constitutive relations $D^{\alpha}=\varepsilon^{\alpha}{ }_{\beta} E^{\beta}$ and $H^{\alpha}=\mu^{\alpha}{ }_{\beta} B^{\beta}$. The coefficients $\varepsilon^{\alpha}{ }_{\beta}=\varepsilon^{\alpha}{ }_{\beta}\left(E^{\mu}, B^{\mu}\right)$ and $\mu^{\alpha}{ }_{\beta}=\mu^{\alpha}{ }_{\beta}\left(E^{\mu}, B^{\mu}\right)$ represent the dielectric tensors. They are usually denoted as permittivity and permeability tensors, respectively. All the information about the dielectric properties of the medium can be encompassed in it.

The propagation of the electromagnetic waves in the eikonal approximation of electrodynamics [4] is obtained by making use of the method of field disturbances $[29,30]$. With the notation introduced before [2] we set $\left[E^{\mu}{ }_{, \nu}\right]_{\Sigma}=$ $e^{\mu} K_{\nu}$ and $\left[B^{\mu}{ }_{, \nu}\right]_{\Sigma}=b^{\mu} K_{\nu}$, where $e^{\mu}$ and $b^{\mu}$ are related to the derivatives of the electric and magnetic fields on the surface of discontinuity $\Sigma$, and they are associated with the polarization of the propagating waves. The quantity $K_{\lambda}=$ $\partial \Sigma / \partial x^{\lambda}$ is the wave 4-vector normal to $\Sigma$. Applying these boundary conditions to the field equations (1) and (2), we obtain the eigenvalue equation [6]

$$
Z^{\alpha}{ }_{\tau} e^{\tau}=0,
$$

where the Fresnel tensor $Z^{\alpha}{ }_{\tau}$ is given by

$$
\begin{aligned}
Z_{\tau}^{\alpha} \doteq & C^{\alpha}{ }_{\tau}+\frac{1}{\omega} \frac{\partial \varepsilon^{\alpha}{ }_{\beta}}{\partial B^{\chi}} \eta^{\chi \omega \gamma}{ }_{\tau} E^{\beta} V_{\gamma} q_{\omega} \\
& +\frac{1}{\omega} \frac{\partial \mu^{\delta}{ }_{\lambda}}{\partial E^{\tau}} \eta^{\alpha \beta \gamma}{ }_{\delta} B^{\lambda} V_{\gamma} q_{\beta}+\frac{1}{\omega^{2}}\left(q^{2} H_{\omega}{ }^{\alpha} I^{\omega}{ }_{\tau}\right. \\
& \left.-q^{2} H_{\chi}{ }^{\chi} I^{\alpha}{ }_{\tau}+H_{\tau}{ }^{\beta} q_{\beta} q^{\alpha}-H_{\chi}{ }^{\delta} q^{\chi} q_{\delta} h^{\alpha}{ }_{\tau}\right),
\end{aligned}
$$

with $\omega \doteq K^{\alpha} V_{\alpha}$ representing the angular frequency of the electromagnetic wave. Additionally, the following definitions are introduced:

$$
\begin{gathered}
h^{\alpha}{ }_{\tau} \doteq \delta^{\alpha}{ }_{\tau}-V^{\alpha} V_{\tau}, \\
q^{\alpha} \doteq h^{\alpha}{ }_{\tau} K^{\tau}=K^{\alpha}-\omega V^{\alpha}, \\
q^{2}=-q^{\alpha} q_{\alpha}=\omega^{2}-K^{2},
\end{gathered}
$$

$$
\begin{gathered}
C^{\alpha}{ }_{\tau} \doteq \varepsilon^{\alpha}{ }_{\tau}+\frac{\partial \varepsilon^{\alpha}{ }_{\beta}}{\partial E^{\tau}} E^{\beta}, \\
H^{\alpha}{ }_{\tau} \doteq \mu^{\alpha}{ }_{\tau}+\frac{\partial \mu^{\alpha}{ }_{\beta}}{\partial B^{\tau}} B^{\beta}, \\
I_{\tau}^{\alpha} \doteq h^{\alpha}{ }_{\tau}+\frac{q^{\alpha} q_{\tau}}{q^{2}} .
\end{gathered}
$$

The general solution for the wave propagation can be derived from the eigenvalue problem stated by the generalized Fresnel equation [Eq. (3)], and is formally given by $\operatorname{det}\left|Z^{\alpha}{ }_{\beta}\right|=0$. There are different ways to solve this problem. In the next section an expansion of the polarization vector $e^{\mu}$ in a suitable basis of linearly independent vectors will be considered in order to obtain solutions from the eigenvalue equation.

\section{THE DISPERSION RELATIONS}

We shall examine the wave propagation issue in material media with dielectric coefficients depending on the intensity of the external fields. For this case we set

$$
\begin{gathered}
\varepsilon^{\alpha}{ }_{\tau}=\varepsilon(E, B) h^{\alpha}{ }_{\tau}, \\
\mu^{\alpha}{ }_{\tau}=\mu^{-1}(E, B) h^{\alpha}{ }_{\tau} .
\end{gathered}
$$

Since these coefficients do not depend on the directions of the external fields, the results are applicable to the study of wave propagation in isotropic liquid media. The application of the results to crystalline structures can be done by considering the explicit dependence of $E^{\mu}$ or $B^{\mu}$ vector fields on the dielectric coefficients. These cases were partially described in $[2,6]$.

From Eqs. (11) and (12), we obtain

$$
\begin{array}{ccc}
\frac{\partial \varepsilon_{\beta}^{\alpha}}{\partial E^{\tau}}=-\varepsilon^{\prime} h_{\beta}^{\alpha} E_{\tau}, & \frac{\partial \varepsilon_{\beta}^{\alpha}}{\partial B^{\tau}}=-\dot{\varepsilon} h_{\beta}^{\alpha} B_{\tau}, \\
\frac{\partial \mu_{\beta}^{\alpha}}{\partial E^{\tau}}=+\frac{\mu^{\prime}}{\mu^{2}} h_{\beta}^{\alpha} E_{\tau}, & \frac{\partial \mu_{\beta}^{\alpha}}{\partial B^{\tau}}=+\frac{\dot{\mu}}{\mu^{2}} h_{\beta}^{\alpha} B_{\tau},
\end{array}
$$

where we have defined $X^{\prime} \doteq(1 / E) \partial X / \partial E$ and $\dot{X} \doteq$ $(1 / B) \partial X / \partial B$, for any quantity $X$.

By introducing the last results in $Z^{\alpha}{ }_{\beta}$, from Eq. (4), it yields

$$
\begin{aligned}
Z^{\alpha}{ }_{\tau}= & {\left[\varepsilon-\frac{q^{2}}{\mu \omega^{2}}-\frac{\dot{\mu} q^{2}}{\mu^{2} \omega^{2}}(B I B)\right] h^{\alpha}{ }_{\tau}-\varepsilon^{\prime} E^{\alpha} E_{\tau} } \\
& +\frac{1}{\omega^{2}}\left(\frac{\dot{\mu} B^{2}}{\mu^{2}}-\frac{1}{\mu}\right) q^{\alpha} q_{\tau}+\frac{\dot{\mu} q^{2}}{\mu^{2} \omega^{2}} B^{\alpha} B_{\tau} \\
& +\frac{\dot{\mu}}{\mu^{2} \omega^{2}}(q B) B^{\alpha} q_{\tau}+\frac{\dot{\mu}}{\mu^{2} \omega^{2}}(q B) q^{\alpha} B_{\tau} \\
& -\left[\frac{\dot{\varepsilon}}{\omega} \eta^{\beta \gamma \delta}{ }_{\tau} E^{\alpha}+\frac{\mu^{\prime}}{\omega \mu^{2}} \eta^{\beta \gamma \delta \alpha} E_{\tau}\right] q_{\beta} V_{\gamma} B_{\delta}
\end{aligned}
$$


where the following additional notation was introduced:

$$
(X I Y) \doteq X_{\mu} I^{\mu \nu} Y_{\nu}=(X Y)+\frac{(q X)(q Y)}{q^{2}},
$$

for any quantities $X$ and $Y$.

In the present form, it is clear that $Z^{\alpha}{ }_{\tau}$ is a 3-dimensional object. Particularly we note the $Z^{\alpha}{ }_{\tau} V^{\tau}=0$. In order to find solutions of the eigenvalue equation, Eq. (3) with $Z^{\alpha}{ }_{\tau}$ given by Eq. (15), we shall consider the expansion of the polarization vector $e^{\tau}$ in a convenient basis of the 3dimensional space as

$$
e^{\tau}=a E^{\tau}+b B^{\tau}+c q^{\tau} .
$$

In this case, from Eqs. (3) and (15), we obtain

$$
\begin{aligned}
& \left\{a\left[\varepsilon-\frac{q^{2}}{\mu \omega^{2}}-\frac{\dot{\mu} q^{2}}{\mu^{2} \omega^{2}}(B I B)+\varepsilon^{\prime} E^{2}-\frac{\dot{\varepsilon}}{\omega} \eta^{\beta \gamma \delta \tau} q_{\beta} V_{\gamma} B_{\delta} E_{\tau}+\frac{\alpha \mu^{\prime} E^{2}}{\omega \mu^{2}}\right]+b\left[-\varepsilon^{\prime}(E B)-\frac{\alpha \mu^{\prime}(E B)}{\omega \mu^{2}}\right]\right. \\
& \left.+c\left[-\varepsilon^{\prime}(q E)-\frac{\alpha \mu^{\prime}(q E)}{\omega \mu^{2}}\right]\right\} E^{\alpha}+\left\{a\left[\frac{\dot{\mu} q^{2}(E B)}{\omega^{2} \mu^{2}}+\frac{\dot{\mu}(q B)(q E)}{\omega^{2} \mu^{2}}+\frac{\beta \mu^{\prime} E^{2}}{\omega \mu^{2}}\right]+b\left[\varepsilon-\frac{q^{2}}{\mu \omega^{2}}-\frac{\beta \mu^{\prime}(E B)}{\omega \mu^{2}}\right]\right. \\
& \left.+c\left[-\frac{\beta \mu^{\prime}(q E)}{\omega \mu^{2}}\right]\right\} B^{\alpha}+\left\{a\left[\frac{1}{\omega^{2}}\left(\frac{\dot{\mu} B^{2}}{\mu^{2}}-\frac{1}{\mu}\right)(q E)+\frac{\dot{\mu}(q B)(E B)}{\omega^{2} \mu^{2}}+\frac{\gamma \mu^{\prime} E^{2}}{\omega \mu^{2}}\right]+b\left[\frac{1}{\omega^{2}}\left(\frac{\dot{\mu} B^{2}}{\mu^{2}}-\frac{1}{\mu}\right)(q B)\right.\right. \\
& \left.\left.\quad-\frac{\dot{\mu} B^{2}(q B)}{\omega^{2} \mu^{2}}-\frac{\gamma \mu^{\prime}(E B)}{\omega \mu^{2}}\right]+c\left[\varepsilon-\frac{\gamma \mu^{\prime}(q E)}{\omega \mu^{2}}\right]\right\} q^{\alpha}=0,
\end{aligned}
$$

where $\alpha, \beta$, and $\gamma$ are given by

$$
\begin{gathered}
\alpha=\frac{q(B I B)}{\sqrt{(E I E)(B I B)-(E I B)^{2}}}, \\
\beta=\frac{-q(E I B)}{\sqrt{(E I E)(B I B)-(E I B)^{2}}}, \\
\gamma=\frac{(q E)(B I B)-(q B)(E I B)}{q \sqrt{(E I E)(B I B)-(E I B)^{2}}} .
\end{gathered}
$$

Since $\left\{E^{\alpha}, B^{\alpha}, q^{\alpha}\right\}$ are taken to be linearly independent vectors, we obtain the solution to the above system by setting each of its coefficients to zero. With a convenient notation, it yields the following system of algebraic equations:

$$
\begin{aligned}
& a\left\{A_{1}\right\}-b\left\{B_{1}\right\}-c\left\{C_{1}\right\}=0, \\
& a\left\{A_{2}\right\}+b\left\{B_{2}\right\}-c\left\{C_{2}\right\}=0, \\
& a\left\{A_{3}\right\}-b\left\{B_{3}\right\}+c\left\{C_{3}\right\}=0,
\end{aligned}
$$

where

$$
\begin{aligned}
A_{1} \doteq & \varepsilon+\left(\varepsilon^{\prime}+\frac{\alpha \mu^{\prime}}{\omega \mu^{2}}\right) E^{2}-\frac{q^{2}}{\mu \omega^{2}}\left[1+\frac{\dot{\mu}}{\mu}(B I B)\right] \\
& -\frac{\dot{\varepsilon}}{\omega}[q V B E], \\
A_{2} \doteq & \frac{\dot{\mu} q^{2}(E I B)}{\omega^{2} \mu^{2}}+\frac{\beta \mu^{\prime} E^{2}}{\omega \mu^{2}}, \\
A_{3} \doteq & \frac{\dot{\mu}}{\omega^{2} \mu^{2}}\left[B^{2}(q E)+(q B)(E B)\right]-\frac{(q E)}{\mu \omega^{2}}+\frac{\gamma \mu^{\prime} E^{2}}{\omega \mu^{2}},
\end{aligned}
$$

$$
\begin{aligned}
& B_{1} \doteq(E B)\left(\varepsilon^{\prime}+\frac{\alpha \mu^{\prime}}{\omega \mu^{2}}\right) \\
& B_{2} \doteq \varepsilon-\frac{q^{2}}{\mu \omega^{2}}-\frac{\beta \mu^{\prime}(E B)}{\omega \mu^{2}}, \\
& B_{3} \doteq \frac{(q B)}{\mu \omega^{2}}+\frac{\gamma \mu^{\prime}(E B)}{\omega \mu^{2}} \\
& C_{1} \doteq(q E)\left(\varepsilon^{\prime}+\frac{\alpha \mu^{\prime}}{\omega \mu^{2}}\right) \\
& C_{2} \doteq \frac{\beta \mu^{\prime}(q E)}{\omega \mu^{2}} \\
& C_{3} \doteq \varepsilon-\frac{\gamma \mu^{\prime}(q E)}{\omega \mu^{2}}
\end{aligned}
$$

with $[q V B E] \doteq \eta^{\alpha \beta \gamma \delta} q_{\alpha} V_{\beta} B_{\gamma} E_{\delta}=\vec{q} \cdot(\vec{E} \times \vec{B})$.

The above set of algebraic equations [Eqs. (22)-(24)] can be solved immediately, resulting in

$$
\begin{gathered}
\left(A_{3} B_{2}+A_{2} B_{3}\right) C_{1}+\left(A_{3} B_{1}-A_{1} B_{3}\right) C_{2} \\
+\left(A_{1} B_{2}+A_{2} B_{1}\right) C_{3}=0 .
\end{gathered}
$$

This is the general equation governing the phenomenon of electromagnetic wave propagation inside material media described by the dielectric coefficients given by Eqs. (11) and (12). Such an equation is usually called the dispersion relation. In the next section some electromagnetic-optic effects will be derived from it and known results concerning birefringence phenomena will be recovered and discussed.

For several physical configurations, the dispersion relations can be presented in the suggestive form $g_{ \pm}^{\mu \nu} K_{\mu} K_{\nu}=$ 0 . The symmetric tensors $g_{ \pm}^{\mu \nu}$ represent the optic metrics 
and, generally, present a solution for each possible polarization mode inside the medium. The integral curves of the vector $K_{\mu}$ are geodesics in the associated effective geometry [3].

Before closing this section some comments on the applicability of the vector basis introduced in Eq. (17) are in order. In the next section we shall apply the results obtained here to study some limiting cases in which two of the vectors used in the basis are parallel. In fact, even for those cases the dispersion relation stated by Eq. (34) holds. It can be understood as follows. Let us consider, for instance, a specific configuration where the angle $\epsilon$ between the vectors $\hat{E}$ and $\hat{B}$ is small. Thus, the products between these vectors can be presented as $\hat{E} \cdot \hat{B} \simeq 1-\epsilon^{2} / 2$ and $\|\hat{E} \times \hat{B}\| \simeq \epsilon$. For $\epsilon \neq 0$ the set of vectors introduced in Eq. (17) will still be a set of basis vectors. Now, if we take $\epsilon$ to be sufficiently small, such that its contribution in the dispersion relation is not measurable, the obtained results must be the same as if $\hat{E}$ and $\hat{B}$ would be parallel vectors. In this way, the results derived in this section can be naturally extended to deal with these limiting cases.

In fact, the dispersion relation obtained in this section can also be obtained without taking in consideration a specific vector basis. Using the Cayley-Hamilton method (see, for instance, Ref. [31]), the eigenvalue equation can be solved by means of the covariant form for the determinant of a three-dimensional matrix as $\left(Z_{1}\right)^{3}-3 Z_{1} Z_{2}+$ $2 Z_{3}=0$, where $Z_{i}(i=1,2,3)$ represents the traces of tensor $Z^{\mu}{ }_{\nu}$. For simple cases, as occur in the derivation of the Kerr effect, the use of the Cayley-Hamilton method is convenient. Nevertheless, for more elaborate situations, as it occurs in the magnetoelectric birefringences, the use of this method leads to laborious calculations. As simple application of this method the Kerr effect is derived in the appendix.

\section{APPLICATIONS TO BIREFRINGENCE PHENOMENA}

In this section some specific examples will be derived from the general dispersion relation presented in Eq. (34). First we will recover some well-known cases, as the Kerr electro-optical and the Cotton-Mouton magneto-optical effects, in which the birefringence phenomena appear as a consequence of the applied external fields. After we will describe the recently measured $[25,26]$ magnetoelectric birefringence. In particular, the anisotropic behavior of the extraordinary ray with respect to the direction of propagation will be carefully examined.

\section{A. Electro and magneto-optical effects}

Let us now consider the particular case where $\varepsilon=\varepsilon(E)$ and $\mu=\mu(B)$. These functional dependences of the dielectric coefficients are, respectively, the necessary conditions to the existence of the Kerr and the Cotton-Mouton birefringence effects in material media, as described by the present formalism.

For this case, it follows from Eq. (34) together with definitions stated in Eqs. (25)-(33), that

$$
\Lambda_{1} v^{4}+\Lambda_{2} v^{2}+\Lambda_{3}=0,
$$

where we have defined the phase velocity $v^{2}=\omega^{2} / q^{2}$, and

$$
\begin{gathered}
\Lambda_{1} \doteq \varepsilon^{2}\left(\varepsilon+\varepsilon^{\prime} E^{2}\right), \\
\Lambda_{2} \doteq \frac{\varepsilon \varepsilon^{\prime} \dot{\mu}}{\mu^{2}}\left[B^{2}(\hat{q} E)^{2}+(E B)^{2}+2(E B)(\hat{q} E)(\hat{q} B)\right] \\
-\frac{\varepsilon^{2} \dot{\mu}(B I B)}{\mu^{2}}-\frac{2 \varepsilon^{2}+\varepsilon \varepsilon^{\prime} E^{2}+\varepsilon \varepsilon^{\prime}(\hat{q} E)^{2}}{\mu}, \\
\Lambda_{3} \doteq \frac{\varepsilon^{\prime} \dot{\mu}(B I B)(\hat{q} E)^{2}+\varepsilon \dot{\mu}(B I B)}{\mu^{3}}+\frac{\varepsilon+\varepsilon^{\prime}(\hat{q} E)^{2}}{\mu^{2}} .
\end{gathered}
$$

Finally, we obtain the following quadratic equation for the phase velocity:

$$
v^{2}=\frac{-\Lambda_{2} \pm \sqrt{\Lambda_{2}^{2}-4 \Lambda_{1} \Lambda_{3}}}{2 \Lambda_{1}} .
$$

In general, we can obtain two solutions from the above equation, which correspond to the two possible polarization modes propagating in the medium.

\section{Kerr birefringence}

By considering $\mu=\mu_{c}=$ constant in Eq. (39) we obtain the following solutions:

$$
v_{o}^{2}=\frac{1}{\mu_{c} \varepsilon}
$$

and

$$
v_{e}^{2}=\frac{1}{\mu_{c}\left(\varepsilon+\varepsilon^{\prime} E^{2}\right)}\left[1+\frac{E}{\varepsilon} \frac{\partial \varepsilon}{\partial E}(\hat{q} \cdot \hat{E})^{2}\right],
$$

where the index $o$ stands for the ordinary ray (o-ray), which propagates isotropically, and the index $e$ stands for the extraordinary ray ( $e$-ray), which depends on the direction of wave propagation. Particularly, if the propagation occurs parallel to the external electric field, $(\hat{q} \hat{E})=$ $\hat{q} \cdot \hat{E}=1$, the velocities of both rays coincide. The difference between them achieves its maximum value when $(\hat{q} \hat{E})=\hat{q} \cdot \hat{E}=0$. In this case Eq. (41) results in

$$
v_{e \perp}^{2}=\frac{1}{\mu_{c}\left(\varepsilon+\varepsilon^{\prime} E^{2}\right)} .
$$

By considering the expansion of the permittivity as

$$
\varepsilon=\varepsilon_{c}+\varepsilon_{1} E^{2},
$$

with $\varepsilon_{c}$ and $\varepsilon_{1}$ constants, we obtain 


$$
\begin{gathered}
v_{e \|}^{2}=v_{o}^{2}=\frac{1}{\mu_{c} \varepsilon_{c}\left(1+\varepsilon_{1} E^{2} / \varepsilon_{c}\right)}, \\
v_{e \perp}^{2}=\frac{1}{\mu_{c} \varepsilon_{c}\left(1+3 \varepsilon_{1} E^{2} / \varepsilon_{c}\right)} .
\end{gathered}
$$

It is assumed that $\varepsilon_{1} E^{2}$ corresponds to a small correction compared to the background permittivity $\varepsilon_{c}$. The corresponding refraction indexes $(n=1 / v)$ are given by

$$
\begin{gathered}
n_{\|}=\frac{1}{v_{e \|}} \simeq \sqrt{\varepsilon_{c} \mu_{c}}\left(1+\frac{\varepsilon_{1} E^{2}}{2 \varepsilon_{c}}\right), \\
n_{\perp}=\frac{1}{v_{e \perp}} \simeq \sqrt{\varepsilon_{c} \mu_{c}}\left(1+\frac{3 \varepsilon_{1} E^{2}}{2 \varepsilon_{c}}\right) .
\end{gathered}
$$

Finally the observable quantity defined as the maximum difference between the refraction indexes in the system is given, up to second order corrections, by

$$
n_{\perp}-n_{\|}=\sqrt{\varepsilon_{c} \mu_{c}} \frac{\varepsilon_{1} E^{2}}{\varepsilon_{c}}
$$

This result is known as the Kerr electro-optic effect. For this case, the presence of an external magnetic field does not produce any change in the results.

\section{Cotton-Mouton birefringence}

Now, taking $\varepsilon=\varepsilon_{c}=$ constant in Eq. (39), we obtain the following solutions:

$$
v_{o}^{2}=\frac{1}{\varepsilon_{c} \mu}
$$

and

$$
v_{e}^{2}=\frac{1}{\varepsilon_{c} \mu}\left[1+\frac{\dot{\mu}}{\mu}(B I B)\right] .
$$

As before, the velocity of the $e$-ray depends on the direction of propagation. In the particular case where the propagation occurs parallel or antiparallel to the external magnetic field $(\hat{q} \cdot \hat{B}= \pm 1)$, its velocity reduces to the velocity of the $o$-ray. The difference between the velocities of the ordinary and extraordinary rays achieve its maximum value when $\hat{q} \cdot \hat{B}=0$. In this case, from Eq. (50),

$$
v_{e \perp}^{2}=\frac{1}{\varepsilon_{c} \mu}\left(1-\frac{\dot{\mu}}{\mu} B^{2}\right) .
$$

By considering the expansion of the permeability as

$$
\mu=\mu_{c}+\varepsilon_{2} B^{2},
$$

with $\mu_{c}$ and $\varepsilon_{2}$ constants, we obtain

$$
v_{e \|}^{2}=v_{o}^{2}=\frac{1}{\mu_{c} \varepsilon_{c}}\left(1-\frac{\varepsilon_{2} B^{2}}{\mu_{c}}\right),
$$

$$
v_{e \perp}^{2}=\frac{1}{\mu_{c} \varepsilon_{c}}\left(1-\frac{3 \varepsilon_{2} B^{2}}{\mu_{c}}\right) \text {. }
$$

By considering $\varepsilon_{2} B^{2} \ll \mu_{c}$, the corresponding refraction indexes are given by

$$
\begin{aligned}
& n_{\|} \simeq \sqrt{\varepsilon_{c} \mu_{c}}\left(1+\frac{\varepsilon_{2} B^{2}}{2 \mu_{c}}\right), \\
& n_{\perp} \simeq \sqrt{\varepsilon_{c} \mu_{c}}\left(1+\frac{3 \varepsilon_{2} B^{2}}{2 \mu_{c}}\right) .
\end{aligned}
$$

Now the difference between these refraction indexes is given by

$$
n_{\perp}-n_{\|} \simeq \sqrt{\varepsilon_{c} \mu_{c}} \frac{\varepsilon_{2} B^{2}}{\mu_{c}} .
$$

This result is known as the Cotton-Mouton magneto-optic effect. The presence of an external electric field is not important for this case.

These two effects, Kerr and Cotton-Mouton birefringences, are really symmetric effects. This symmetry is related to the fact that the Maxwell equations in the absence of sources are symmetric with respect to the duality rotation.

\section{B. Magnetoelectric birefringence}

As another application of the present formalism, let us now examine some features of the so-called magnetoelectric birefringence. For simplicity, we shall assume in this section the particular case where $\varepsilon=\varepsilon(E, B)$ and $\mu=$ $\mu_{c}=$ constant.

In this case, from Eq. (34) we obtain the following solutions for the phase velocities of both the ordinary and the extraordinary rays:

$$
v_{o}= \pm \frac{1}{\sqrt{\mu_{c} \varepsilon}}
$$

and

$$
v_{e}^{ \pm}=\frac{\dot{\varepsilon}[\hat{q} V B E]}{2\left(\varepsilon+\varepsilon^{\prime} E^{2}\right)} \pm \sqrt{\frac{\dot{\varepsilon}^{2}[\hat{q} V B E]^{2}}{4\left(\varepsilon+\varepsilon^{\prime} E^{2}\right)^{2}}+\frac{\varepsilon+\varepsilon^{\prime}(\hat{q} E)^{2}}{\mu \varepsilon\left(\varepsilon+\varepsilon^{\prime} E^{2}\right)}} .
$$

As one can see, the equation for the velocity of the $e$-ray contains two possible solutions, which are not obviously symmetric with respect to the direction of propagation. Together with the $o$-ray we generally have three distinct solutions for the phase velocities. In fact the two solutions associated with the $e$-ray indicate that it can present distinct velocities in opposite directions. This aspect will be further addressed in the analysis of some particular cases.

Let us consider the following expansion for the permittivity coefficient:

$$
\varepsilon=\varepsilon_{c}+\varepsilon_{1} E^{2}+\varepsilon_{2} B^{2}+\varepsilon_{3}(\vec{E} \cdot \vec{B}) .
$$


For practical cases, the terms in $\varepsilon_{i}(i=1,2,3)$ represent just small corrections to the main term $\varepsilon_{c}$-the background permittivity. By using this expansion we obtain the following results for the velocities of the above rays, up to second order terms in $\varepsilon_{i}$,

$$
v_{o}= \pm \frac{1}{\sqrt{\varepsilon_{c} \mu_{c}}}\left[1-\frac{\varepsilon_{1}}{2 \varepsilon_{c}} E^{2}-\frac{\varepsilon_{2}}{2 \varepsilon_{c}} B^{2}-\frac{\varepsilon_{3}}{2 \varepsilon_{c}} E B(\hat{E} \cdot \hat{B})\right]
$$

and

$$
\begin{aligned}
v_{e}^{ \pm}= & {\left[\frac{\varepsilon_{2}}{\varepsilon_{c}} E B+\frac{\varepsilon_{3}}{2 \varepsilon_{c}} E^{2}(\hat{E} \cdot \hat{B})\right] \hat{q} \cdot(\hat{E} \times \hat{B}) } \\
& \pm \frac{1}{\sqrt{\varepsilon_{c} \mu_{c}}}\left\{1+\left[(\hat{q} \cdot \hat{E})^{2}-\frac{3}{2}\right] \frac{\varepsilon_{1}}{\varepsilon_{c}} E^{2}-\frac{\varepsilon_{2}}{2 \varepsilon_{c}} B^{2}\right. \\
& \left.+\frac{\varepsilon_{3}}{2 \varepsilon_{c}} E B(\hat{E} \cdot \hat{B})\left[(\hat{q} \cdot \hat{E})^{2}-2\right]\right\} .
\end{aligned}
$$

Now, two particular cases of current experimental interest are analyzed. First we shall derive an example of the recently measured magnetoelectric linear birefringence [26]. After, the so-called Jones effect will be presented. The latter is also a kind of magnetoelectric birefringence which was predicted long ago [21] and was recently measured [25].

\section{Some specific cases}

Let us consider the case where the direction of the wave vector $\hat{q}$ is perpendicular to the plane which contains the electric $\vec{E}$ and magnetic $\vec{B}$ fields. Let us work in a Cartesian coordinate system and set $\hat{E} \cdot \hat{B}=\cos \theta$ and $\hat{q}=\hat{z}$ such that $\hat{q}=(\hat{E} \times \hat{B}) /\|\hat{E} \times \hat{B}\|$. Up to second order terms, the velocity of the $e$-ray, given by Eq. (62), is

$$
v_{e}^{ \pm}=P \pm Q
$$

where

$$
\begin{gathered}
P \doteq\left(\frac{\varepsilon_{2}}{\varepsilon_{c}} E B+\frac{\varepsilon_{3}}{2 \varepsilon_{c}} E^{2} \cos \theta\right) \sin \theta \\
Q \doteq \frac{1}{\sqrt{\varepsilon_{c} \mu_{c}}}\left(1-\frac{3}{2} \frac{\varepsilon_{1}}{\varepsilon_{c}} E^{2}-\frac{\varepsilon_{2}}{2 \varepsilon_{c}} B^{2}-\frac{\varepsilon_{3}}{\varepsilon_{c}} E B \cos \theta\right)
\end{gathered}
$$

Since $\varepsilon_{i}$ refers to small perturbations compared to the background permittivity $\varepsilon_{c}$, we obtain $Q>0$ and $Q>P$. In this way, $v_{e}^{+}>0$ and $v_{e}^{-}<0$. Summarizing, we obtain that $\vec{v}_{e}^{+}=\left|v_{e}^{+}\right| \hat{z}$ and $\vec{v}_{e}^{-}=-\left|v_{e}^{-}\right| \hat{z}$. In other words, when the $e$-ray propagates in the $\hat{z}$ direction it will present a phase velocity which is generally different from the velocity of itself propagating in the opposite direction $(-\hat{z})$. Thus, for each direction $\hat{q}$ there will be only one value for the velocity of the extraordinary ray, which propagates anisotropically. Additionally, since the ordinary ray propagates isotropically, there will be birefringence phenomena in any direction but those where the velocity of both rays coincide.

With the usual definition of the refraction index $(n=$ $1 / v$ ) we obtain the following result for the maximum difference between the propagation of both rays in the present situation:

$$
\begin{aligned}
n_{\|}-n_{\perp} \simeq & \sqrt{\varepsilon_{c} \mu_{c}}\left[-\frac{\varepsilon_{1}}{\varepsilon_{c}} E^{2}+\frac{\varepsilon_{2}}{\varepsilon_{c}} \sqrt{\varepsilon_{c} \mu_{c}} E B \sin \theta\right. \\
& \left.-\frac{\varepsilon_{3}}{2 \varepsilon_{c}}\left(E B-\sqrt{\varepsilon_{c} \mu_{c}} E^{2} \sin \theta\right) \cos \theta\right] .
\end{aligned}
$$

It is interesting to note that, by considering the wave vector in the opposite direction $(-\hat{q})$, the result presented in Eq. (66) is modified as

$$
\begin{aligned}
n_{\|}-n_{\perp} \simeq & \sqrt{\varepsilon_{c} \mu_{c}}\left[-\frac{\varepsilon_{1}}{\varepsilon_{c}} E^{2}-\frac{\varepsilon_{2}}{\varepsilon_{c}} \sqrt{\varepsilon_{c} \mu_{c}} E B \sin \theta\right. \\
& \left.-\frac{\varepsilon_{3}}{2 \varepsilon_{c}}\left(E B+\sqrt{\varepsilon_{c} \mu_{c}} E^{2} \sin \theta\right) \cos \theta\right] .
\end{aligned}
$$

In this case [Eq. (67)] the terms in $\varepsilon_{1}$ and $\varepsilon_{2}$ present the same sign. Thus, if $\varepsilon_{1} / \varepsilon_{2}>0$ and $\theta=\pi / 2$ in the latter configuration of fields, the magnetoelectric contribution to the birefringence would magnify the effect.

a. Crossed fields: The case of crossed electric and magnetic fields $(\theta=\pi / 2)$, which has been experimentally measured, is obtained directly from Eq. (66) as

$$
n_{\|}-n_{\perp} \simeq \sqrt{\varepsilon_{c} \mu_{c}}\left(-\frac{\varepsilon_{1}}{\varepsilon_{c}} E^{2}+\frac{\varepsilon_{2}}{\varepsilon_{c}} \sqrt{\varepsilon_{c} \mu_{c}} E B\right) .
$$

The term corresponding to the Cotton-Mouton effect does not appear since we have considered the particular case of nonmagnetic medium $\left(\mu=\mu_{c}=\right.$ constant). The inclusion of this term can be done simply by setting the permeability as a function of the magnetic field.

A kind of magnetoelectric birefringence will still occur when the medium behaves as $\varepsilon=\varepsilon(B)$ and both external fields $\vec{E}$ and $\vec{B}$ are present. In this case Eq. (68) reduces to $n_{\|}-n_{\perp} \simeq \mu_{c} \varepsilon_{2} E B$, which shows that the magnetoelectric birefringence would be the dominant effect. In fact, it appears as the unique effect. This nonstandard magnetoelectric birefringence occurs also in the more general situation of noncrossed fields. In this case, the difference between the refraction indexes is given by

$$
n_{\|}-n_{\perp} \simeq \mu_{c} \varepsilon_{2} E B \sin \theta .
$$

We notice that its maximum value occurs when the fields are crossed $(\theta=\pi / 2)$.

$b$. Jones birefringence: The Jones effect is another kind of magnetoelectric birefringence, also recently observed [25], which occurs with parallel electric $\vec{E}$ and magnetic $\vec{B}$ fields perpendicular to the wave vector $\vec{q}$. This case can be obtained directly from Eq. (66) [or from Eq. (67)] and results in 


$$
n_{\|}-n_{\perp} \simeq \sqrt{\varepsilon_{c} \mu_{c}}\left(-\frac{\varepsilon_{1}}{\varepsilon_{c}} E^{2}-\frac{\varepsilon_{3}}{2 \varepsilon_{c}} E B\right) .
$$

Magnetoelectric birefringence with crossed fields and Jones birefringence are very similar effects. Nevertheless, they are independent to each other. Note that the terms in the product $E B$ are proportional to different coefficients. In the case of crossed fields it is proportional to the coefficient $\varepsilon_{2}$, which is coupled with the dependence on $B^{2}$ in the permittivity. On the other hand, in the case of Jones birefringence, it is proportional to the coefficient $\varepsilon_{3}$, coupled with the dependence on the product $E B$ in the permittivity.

Another interesting aspect related to these effects is the form of the front wave at a given instant of time. As we have shown, for all cases the $e$-ray propagates anisotropically. Particularly, the magnitude of the effect is generally different in opposite directions. In order to visualize the effect of this anisotropy in the propagation direction, let us analyze a particular case where $(\hat{E} \hat{B})=0,(\hat{q} \hat{B})=0$, and $(\hat{q} \hat{E})=\cos \psi$. Let us consider a Cartesian coordinate system such that $\hat{E}=\hat{x}$ and $\hat{B}=\hat{y}$ and solve the equation for the curves representing the intersection of the normal surfaces with the $X Z$-plane. Since the $o$-ray propagates isotropically, its associated normal surface is represented by a circle in this plane, at any instant of time. On the other

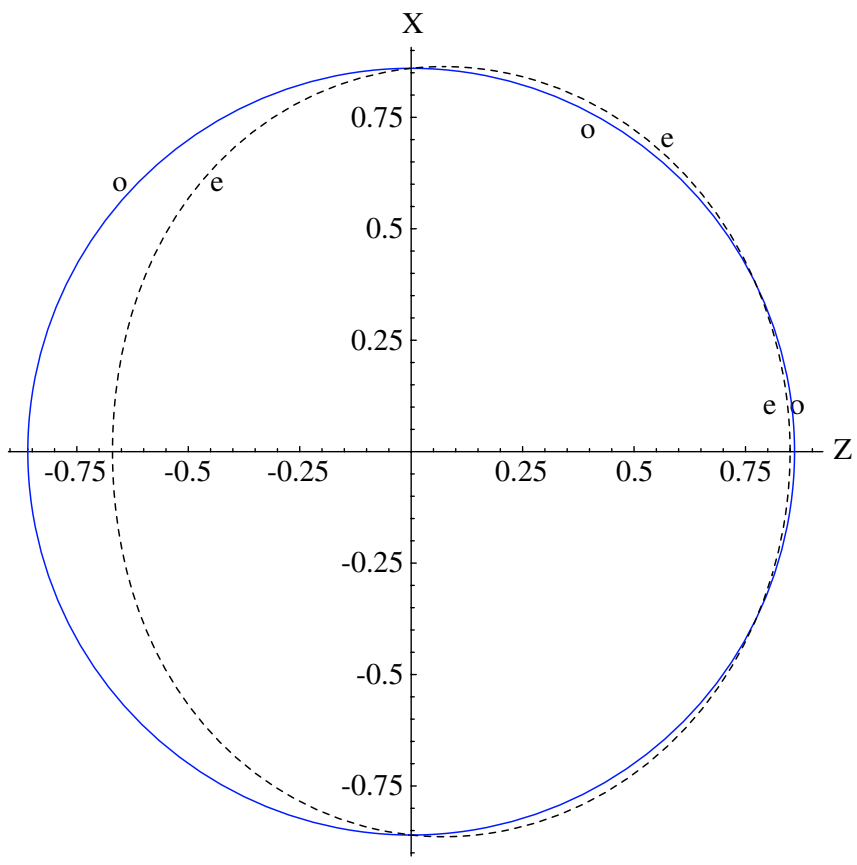

FIG. 1 (color online). Normal surfaces for the ordinary (circular solid line) and extraordinary (dashed line) rays propagating in an isotropic material media with dielectric coefficients given by $\varepsilon=\varepsilon(E, B)$ and $\mu=\mu_{c}=$ constant. The symbols $e$ and $o$ stand for the front waves associated with the $e$-ray and $o$-ray, respectively. The plot is based on Eqs. (61) and (62), where we have considered the field configuration as $\hat{E}=\hat{x}$ and $\hat{B}=\hat{y}$, and also $\hat{q} \cdot \hat{B}=0$. The numerical values were chosen as to satisfy the approximations assumed in Sec. IV B. hand, the $e$-ray does not propagate isotropically and its normal surface will be not symmetric with respect to the plane $X Y$, as shown in Fig. 1. We notice the possibility that the velocity of the $e$-ray be greater or smaller than the velocity of the $o$-ray, depending on the direction of propagation. Further, depending on the parameters in the permittivity expansion, there will be more than two directions in which the velocities of both rays coincide.

For any of the cases considered in this section the corresponding polarization vectors $e^{\mu}$ can be directly obtained by returning each specific solution in the set of equations (22)-(24) and solving it for the coefficients $a$, $b$, and $c$.

\section{CONCLUSION}

A tensorial formalism was here presented for the description of monochromatic electromagnetic waves inside material media with nonlinear dielectric properties. The limit of geometrical optics was considered. The eigenvector problem for general media was presented and solved for isotropic material media characterized by dielectric coefficients as $\varepsilon_{\mu \nu}(E, B)$ and $\mu_{\mu \nu}(E, B)$. The solution for the wave propagation in isotropic media described by these coefficients was obtained directly from the general dispersion relation expressed by Eq. (34). Using this main result, a detailed analysis of the birefringence phenomena was performed. Some well-known effects were recovered and some new information was presented. The results include the description of the Kerr electro-optical effect, the Cotton-Mouton magneto-optical effect, and the recently measured magneto-electric birefringences.

It was shown that the Kerr birefringence in isotropic media is fundamentally related to the dependence of the permittivity on the electric field as $\varepsilon(E)$. The application of an external magnetic field does not bring changes to the effect. Symmetrically, the necessary condition for the appearance of the Cotton-Mouton effect is the dependence of the permeability on the magnetic field as $\mu(B)$. Similarly, an applied electric field does not alter the results.

In what refers to the magnetoelectric birefringence, some few remarks are in order. First, from the general dispersion relation there were found three possible solutions for the phase velocities. One is related to the propagation of the ordinary ray and the other two are related to the propagation of the extraordinary ray. The two generally distinct values for the extraordinary ray are related to the distinct values of its velocity in opposite directions, as it was explicitly shown for the case where $\hat{q}$ is perpendicular to the plane that contains the electric and magnetic fields. For the case of parallel fields it was found that the propagation is symmetric in opposite directions. In both cases the propagation occurs anisotropically. Another remarkable result is the birefringence effect occurring with the permittivity $\varepsilon(B)$ and constant permeability $\mu_{c}$, in the presence of external fields. For this case, if the external 
electric field is absent there will be no birefringence. Nevertheless, if both the electric and the magnetic fields are present a kind of magnetoelectric birefringence appears as the unique effect. This result shows that it is possible to produce such a birefringence effect without any other accompanying standard birefringence, as the Kerr or Cotton-Mouton effects. By symmetry, it can be inferred that a similar effect occurs with $\mu(E)$ and constant permittivity. Finally, the behavior of the front wave associated with the extraordinary ray should be stressed. Depending on each medium in which the propagation occurs, the extraordinary ray can present its velocity greater or smaller than the velocity of the ordinary ray depending on the direction of propagation. Further, the coincidence between both rays can occur in several directions.

\section{ACKNOWLEDGMENTS}

The authors are grateful to A. C. Zambroni de Souza for reading the manuscript. This work was partially supported by the Brazilian research agencies CNPq and FAPEMIG. G. P. G. thanks the support from FAPEMIG during his MSc studies.

\section{APPENDIX}

In this appendix, the Kerr effect is derived by means of the Cayley-Hamilton method [31]. Using the same assumptions presented in Sec. IVA 1 we obtain, from Eq. (15),

$$
Z^{\alpha}{ }_{\tau}=\left(\varepsilon-\frac{q^{2}}{\mu_{c} \omega^{2}}\right) h^{\alpha}{ }_{\tau}-\varepsilon^{\prime} E^{\alpha} E_{\tau}-\frac{1}{\mu_{c} \omega^{2}} q^{\alpha} q_{\tau} .
$$

The solutions of the eigenvalue problem stated by Eq. (3) is formally given by $\operatorname{det}\left|Z^{\alpha}{ }_{\tau}\right|=0$, which can be evaluated as

$$
\left(Z_{1}\right)^{3}-3 Z_{1} Z_{2}+2 Z_{3}=0,
$$

with $Z_{i}(i=1,2,3)$ representing the traces

$$
Z_{1} \doteq Z_{\alpha}^{\alpha}=3 \varepsilon-\frac{2 q^{2}}{\mu_{c} \omega^{2}}+\varepsilon^{\prime} E^{2}
$$

$$
\begin{aligned}
Z_{2} \doteq & Z^{\alpha}{ }_{\tau} Z^{\tau}{ }_{\alpha} \\
= & 3\left(\varepsilon-\frac{q^{2}}{\mu_{c} \omega^{2}}\right)^{2}+2\left(\varepsilon^{\prime} E^{2}+\frac{q^{2}}{\mu_{c} \omega^{2}}\right)\left(\varepsilon-\frac{q^{2}}{\mu_{c} \omega^{2}}\right) \\
& +\frac{2 \varepsilon^{\prime}(q E)^{2}}{\mu_{c} \omega^{2}}+\frac{q^{4}}{\mu_{c}^{2} \omega^{4}}+\varepsilon^{\prime 2} E^{4}, \\
Z_{3} \doteq & Z_{{ }^{\alpha} Z{ }^{\tau}{ }_{\beta} Z^{\beta}{ }_{\alpha}} \\
= & 3\left(\varepsilon-\frac{q^{2}}{\mu_{c} \omega^{2}}\right)^{3}+3\left(\varepsilon^{\prime} E^{2}+\frac{q^{2}}{\mu_{c} \omega^{2}}\right)\left(\varepsilon-\frac{q^{2}}{\mu_{c} \omega^{2}}\right)^{2} \\
& +3\left[\varepsilon^{\prime 2} E^{4}+\frac{2 \varepsilon^{\prime}(q E)^{2}}{\mu_{c} \omega^{2}}+\frac{q^{4}}{\mu_{c}^{2} \omega^{4}}\right]\left(\varepsilon-\frac{q^{2}}{\mu_{c} \omega^{2}}\right) \\
& +\varepsilon^{13} E^{6}+\frac{3 \varepsilon^{\prime 2} E^{2}(q E)^{2}}{\mu_{c} \omega^{2}}+\frac{3 \varepsilon^{\prime} q^{2}(q E)^{2}}{\mu_{c}^{2} \omega^{4}}+\frac{q^{6}}{\mu_{c}^{3} \omega^{6}} .
\end{aligned}
$$

Now, introducing the results (A3)-(A5) in Eq. (A2), and using the definitions introduced in Secs. III and IV, we obtain

$$
\left(1-\mu_{c} \varepsilon v^{2}\right)\left[\mu_{c} \varepsilon\left(\varepsilon+\varepsilon^{\prime} E^{2}\right) v^{2}-\varepsilon-\varepsilon^{\prime} E^{2}(\hat{q} \cdot \hat{E})^{2}\right]=0 .
$$

As one can see, the above equation presents two solutions which are the same solutions obtained in Sec. IVA 1 for the ordinary and extraordinary rays, as stated by Eqs. (40) and (41). Now, the Kerr birefringence is obtained in the same lines as discussed in Sec. IVA 1.
[1] M. Schonberg, Revista Brasileira de Fisica 1, 91 (1971); Y. N. Obukhov and F. W. Hehl, Phys. Lett. B 458, 466 (1999); Y. N. Obukhov, T. Fukui, and G. F. Rubilar, Phys. Rev. D 62, 044050 (2000).

[2] V. A. De Lorenci and M. A. Souza, Phys. Lett. B 512, 417 (2001).

[3] V. A. De Lorenci and R. Klippert, Phys. Rev. D 65, 064027 (2002); V. A. De Lorenci, Phys. Rev. E 65, 026612 (2002).

[4] L. Landau and E. Lifchitz, Électrodynamique des Milieux Continus (Mir, Moscou, 1969).

[5] M. Born and E. Wolf, Principles of Optics (Cambridge University Press, Cambridge, England, 1980), 6th ed.

[6] V. A. De Lorenci, R. Klippert, and D. H. Teodoro, Phys. Rev. D 70, 124035 (2004).

[7] W. Heisenberg and H. Euler, Z. Phys. 98, 714 (1936); J.
Schwinger, Phys. Rev. 82, 664 (1951).

[8] Z. Bialynicka-Birula and I. Bialynicki-Birula, Phys. Rev. D 2, 2341 (1970).

[9] S. L. Adler, Ann. Phys. (N.Y.) 67, 599 (1971).

[10] W. Dittrich and H. Gies, Phys. Rev. D 58, 025004 (1998); Phys. Lett. B 431, 420 (1998).

[11] V.A. De Lorenci, R. Klippert, M. Novello, and J. M. Salim, Phys. Lett. B 482, 134 (2000).

[12] M. Novello, V.A. De Lorenci, J. M. Salim, and R. Klippert, Phys. Rev. D 61, 045001 (2000).

[13] G. W. Gibbons and C. A. R. Herdeiro, Phys. Rev. D 63, 064006 (2001).

[14] Y. N. Obukhov and G. F. Rubilar, Phys. Rev. D 66, 024042 (2002).

[15] J. Kerr, Philos. Mag. 50, 337 (1875). 
[16] J. Kerr, Philos. Mag. 50, 416 (1875).

[17] A. Cotton and M. Mouton, Compte Rendu 141, 317 (1905).

[18] A. Cotton and M. Mouton, Compte Rendu 141, 349 (1905).

[19] A. Cotton and M. Mouton, Compte Rendu 145, 229 (1907).

[20] A. Cotton and M. Mouton, Compte Rendu 145, 870 (1907).

[21] R. C. Jones, J. Opt. Soc. Am. 38, 671 (1948).

[22] N. B. Baranova, Yu. V. Bogdanov, and B. Ya. Zel'dovich, Sov. Phys. Usp. 20, 870 (1977).

[23] E. B. Graham and R. E. Raab, Mol. Phys. 52, 1241 (1984).

[24] H. J. Ross, B. S. Sherborne, and G. E. Stedman, J. Phys. B 22, 459 (1989).

[25] T. Roth and G. L. J. A. Rikken, Phys. Rev. Lett. 85, 4478 (2000).
[26] T. Roth and G. L. J. A. Rikken, Phys. Rev. Lett. 88, 063001 (2002).

[27] G. D. Fleishman, Q. J. Fu, M. Wang, G.-L. Huang, and V. F. Melnikov, Phys. Rev. Lett. 88, 251101 (2002); H. J. Mosquera Cuesta, J. A. Freitas Pacheco, and J. M. Salim, Int. J. Mod. Phys. A 21, 43 (2006).

[28] In the absence of sources the Maxwell equations can be represented by the set of equations: $P^{\mu \nu}{ }_{, \mu}=0$ and $\star F^{\mu \nu}{ }_{\mu}=0, \quad$ where $\quad P^{\mu \nu}=V^{\mu} D^{\nu}-V^{\nu} D^{\mu}-$ $\eta^{\mu \nu \alpha \beta} V_{\alpha} H_{\beta}$ and $\star F^{\mu \nu}=V^{\mu} B^{\nu}-V^{\nu} B^{\mu}+\eta^{\mu \nu \alpha \beta} V_{\alpha} E_{\beta}$.

[29] J. Plebanski, in Lectures on Nonlinear Electrodynamics (Nordita, Copenhagen, 1968).

[30] J. Hadamard, in Leçons sur la Propagation des ondes et les équations de l'hydrodynamique (Hermann, Paris, 1903); G. Boillat, J. Math. Phys. (N.Y.) 11, 941 (1970).

[31] R. R. Silva, J. Math. Phys. (N.Y.) 39, 6206 (1998). 